\title{
LE DÉVELOPPEMENT DES RECHERCHES SUR LA RADIO EN AFRIQUE
}

\author{
Étienne Lakétienkoia Damome ${ }^{1}$
}

Média le plus développé, le seul auquel beaucoup de ruraux ont accès, la radio est aussi celui qui s'est le mieux africanisé. Les populations se le sont approprié à cause de son ancrage dans l'oralité et de sa proximité, faisant ainsi revivre à la fois l'ambiance communautaire et la palabre. On devine bien les multiples enjeux dans lesquels s'inscrivent les pratiques radiophoniques et l'intérêt multiforme que les études sur la radio en Afrique peuvent receler. Et pourtant, on ne peut pas dire que l'analyse de la radiophonie en Afrique passionne grand monde. Média le plus populaire, la radio reste le média que les intellectuels prennent le moins en compte dans leurs recherches sur les médias, la presse écrite ayant donné le plus grand nombre d'études.

Après avoir fait un bref historique des études sur la radio en Afrique, nous tracerons quelques pistes de recherches et proposerons

1 Doctorant en Sciences de l'Information et de la Communication, Université de Bordeaux 3

Recherches en communication, $\mathrm{n}^{\circ} 26$ (2006). 
à ceux que cela peut intéresser des orientations pour la localisation des sources.

\section{État des lieux}

L'évolution des études sur la radio peut être analysée selon plusieurs approches. On peut adopter une approche épistémologique, recherchant dans quels champs de recherche ont pu apparaître ces études, comment elles se sont intégrées dans les études sur la communication et sur la communication de masse. On peut également les aborder à partir d'une approche historique présentant l'évolution des études sur la radio en relation avec l'évolution de la radio elle-même, des sociétés dans lesquelles elle s'est implantée. C'est cette deuxième posture que nous privilégions. Nous retraçons l'itinéraire décrit par les études avant de présenter leur contenu.

\section{Petite histoire des recherches sur la radio en Afrique}

Aussi surprenant que cela puisse nous paraître aujourd'hui, les études sur la radio en Afrique sont contemporaines de l'émergence de la radiophonie dans ce continent. En dehors d'un article paru dans le New York Times en mai 1927 sur la « New Radio Station for French Equatorial Africa », la première étude concernant la radio en Afrique remonte à 1937 lorsque Arno G. Huth inclut l'Afrique dans son étude sur La radiodiffusion : puissance mondiale. La radio n'était alors qu'à ses débuts en Afrique. Lorsqu'elle aura conquis toute l'Afrique après la deuxième guerre mondiale, les chercheurs en prendront la mesure. On ne trouve pas moins de 11 études entre 1942 et 1959 concernant directement l'un ou l'autre pays d'Afrique ou traitant de la radio dans le monde en incluant l'Afrique. Le nombre augmentera sensiblement entre les années 1960 et 1970 . Force est de noter que c'est du côté anglophone qu'on trouve un réel plaisir à étudier la radio africaine. À partir de 1960, la radio en Afrique intéressera également toutes sortes d'organisme qui commandent des études. Citons uniquement United States Information Agency (USIA) qui entamera à partir de 1964 un travail gigantesque de rapports sur la radiophonie dans les diverses régions du continent. À intervalle de deux ans, ces rapports abordent 
divers aspects touchant au statut juridique, aux programmes, à la réception des radios, etc. Ce travail aura pour ainsi dire indiqué la voie à Sydney W. Head qui fit le même travail en 1974 à l'échelle de toute l'Afrique continentale et des îles. Broadcasting in Africa, A continental survey of Radio and Television auquel plusieurs Africains ont contribué grandement présente la situation de la radio et aborde à son propos divers aspects (historiques, économiques, politiques, culturels, administratifs...) pays par pays.

Du côté francophone, en dehors de l'initiative de Arno G. Huth en 1937, l'intérêt pour la radio s'est manifesté assez tardivement. Les premières études repérables datent du début des années 1960 au moment où les écoles de journalisme occidentales s'ouvraient aux étudiants africains. Francis Bebey, parmi d'autres, montrera un goût particulier dans l'étude de la radio en Afrique noire. Son livre publié sous le titre de $L a$ radio en Afrique aux éditions St Paul en 1963 constitue aujourd'hui encore l'une des sources confirmées de l'histoire de la radio en Afrique. Mais c'est surtout à partir des années 1970 que les études sur la radio vont prendre une dimension continentale. À cette époque, elles étaient le fait de professionnels ou futurs professionnels réfléchissant sur les différents aspects de leur métier et leurs difficultés afférentes. On trouve en effet, avec la naissance sur place des écoles de journalisme, plusieurs mémoires d'étudiants en fin de cycle qui prennent la radio comme sujet d'analyse. La plupart des études donnent un portrait intéressant des radios publiques (radios d'États) à l'heure du monopole d'exploitation de la radiophonie par l'État. On trouve cependant un certain nombre de travaux de chercheurs occidentaux.

Les années 1990 ont révélé un intérêt plus accru des universitaires et des chercheurs pour la radio. Moins soucieux de décrire la pratique, ou de proposer des solutions à des problèmes ou encore de définir les règles d'une bonne pratique radiophonique, ceux-ci ont abordé des sujets plus théoriques. On peut évoquer plusieurs thèses soutenues sur des sujets divers. Pour la plupart, ce sont des études monographiques qui ne manquent pas d'intérêt mais qui ont la particularité d'aborder des problèmes concrets. C'est avec $\mathrm{M}$. Tudesq, qui a inauguré dans les années 1980 une recherche d'envergure ayant donné lieu plus tard à des ouvrages de référence sur les médias africains, qu'on trouve des études d'ensemble consacrées à la radio. Depuis lors, plusieurs autres 
chercheurs africains et africanistes ont fait de la radio l'objet privilégié de leurs recherches.

Sur place en Afrique, aujourd' hui, il faut déplorer une insuffisance d'intérêt des chercheurs locaux. L'Institut Panos Afrique de l'Ouest, travaillant en collaboration avec les professionnels des différents pays, a produit une abondante documentation sur les médias parmi lesquels certains consacrés à la radio. On ne voit pas cependant beaucoup d'universitaires ou de chercheurs attitrés y collaborer. Cela est d'autant plus surprenant que le paysage radiophonique et même la pratique radiophonique ont subi de profondes mutations ces dernières années. Cette remarque vaut pour toute l'Afrique mais surtout pour l'Afrique francophone qui ne semble pas prendre encore la vraie mesure du phénomène. La radio en Afrique d'aujourd'hui reste donc un champ presque totalement à explorer.

\section{Le contenu des études}

Comme le faisait remarquer André-Jean Tudesq ${ }^{1}$, les études sur la radio évoluent selon les mutations que la radio a connues et connaît encore : mutations techniques qui se propagent de plus en plus vite des pays qui les inventent au reste du monde, mutations des statuts liés à la conjoncture politique propre à chaque État, évolution des contenus et des publics. Ces études se différencient selon qu'elles considèrent la radio comme objet d'étude ou sujet d'étude. Comme objet d'étude, il s'agit surtout des institutions de la radio et de sa législation avec des études juridiques mais également des émissions et des publics. Prenant la radio comme sujet, les études analysent son évolution et son insertion dans la société, les corrélations entre les évolutions techniques et structurelles, les émissions et les publics.

Les études de la radio ne sont pas tributaires seulement de l'évolution de la radio et de la société dans laquelle elle est étudiée, mais aussi de l'évolution des sciences sociales et de leurs méthodes. On peut ainsi entrevoir une approche multidisciplinaire en sciences de l'information

1 André-Jean TUDESQ, « Les études sur la radio et la périodisation de la radio », communication à l'Université d'été de Sienne, été 2004. 
et de la communication, en histoire, en sciences politiques, en linguistique et même en lettres modernes. Les approches ont donné lieu à des questionnements multiples. La pluralité et la diversité des approches font des études existantes des sources très riches pour les investigations actuelles. De grandes tendances peuvent être dégagées qu'il faut relier à l'évolution des sociétés africaines mais aussi à celle de la conception de la radio, des structures et de la technique. Les thématiques varient en fonction des périodes car le sujet lui même s'est révélé extrêmement mouvant. Toutefois, les travaux ont toujours été marqués par leurs contenus concrets.

Le répertoire des études faites révèle cinq préoccupations principales qui se sont succédées presque chronologiquement : la conquête du terrain Africain, la radio et la construction des jeunes nations, la radio comme outil d'éducation et de développement, la radio dans un contexte de pluralisme politique et médiatique, et la radio dans une situation de crise.

\section{7-1980}

Durant la période coloniale, outre la description du paysage radiophonique et des pratiques radiophoniques, l'étude des programmes, les aspects techniques et financiers, les études abordent le rôle des radios dans « la guerre des ondes ». Tous ces thèmes sont annexés à ceux qui ont cours dans les métropoles.

Durant les années 1960, 1970 et 1980, l'intérêt s'est d'abord porté sur l'évaluation des missions que les gouvernants mais aussi les organismes internationaux comme l'UNESCO avaient confiée à la radio en matière de construction des jeunes nations, du renforcement de l'unité nationale, du développement socio-économique ${ }^{1}$, de l'éducation et de la culture. Ainsi les thèmes dominants sont : l'éducation, la santé, l'agriculture, l'alphabétisation, la place des langues locales, le phénomène des radios rurales ${ }^{2}$. À côté de ces thèmes majeurs, beaucoup se sont intéressés aux questions plus générales rentrant dans le cadre habituel

1 K.L. AHONTO, Mass media et développement des jeunes nations : le cas du Togo, 1980.

2 André-Jean TUDESQ, La Radio rurale en Afrique, 1980.

Voir aussi B. FYLLA-SAINT-EUDES, La grille des programmes de la voix de la révolution congolaise et les émissions de la radio rurale 1977-1982, 1989. 
des recherches en communication. Le système institutionnel ${ }^{1}$, l'économie des moyens d'information ${ }^{2}$, les politiques d'informations, les préoccupations historiques, le contenu des programmes, la question des audiences, de la réception et des usages ont ainsi été abordés dans de nombreuses études monographiques et quelques unes d'ensemble (par exemple l'étude des audiences dans plusieurs capitales).

Vers la fin des années 1980 et au début des années 1990, on prit la mesure de l'influence croissante des radios étrangères en Afrique. Cela a donné lieu à des études sur la coopération des pays du Nord en matière de radiophonie, la subrogation croissante des radios africaines par les radios étrangères, la dévalorisation des radios d'État, leur minorisation en tant qu'instrument de pouvoir du fait de l'insertion de l'Afrique dans les circuits mondiaux de la communication ${ }^{3}$.

\section{Des années 1990 à nos jours}

La plupart des thèmes précédents ont été repris dans les années 1990-2000 à cause du réveil tardif de l'intérêt des études sur la radio dans les pays francophones. Plusieurs études se sont focalisées sur l'impact et la réception des radios étrangères ${ }^{4}$, les enjeux culturels de la programmation, etc. Il faut noter cependant que le nouveau contexte médiatique que connaît l'Afrique depuis ce temps oriente différemment les thématiques. Le pluralisme radiophonique ${ }^{5}$ dont il faut rendre compte a ainsi généré beaucoup de monographies sur le paysage radiophonique de nombreux pays ${ }^{6}$. Mais cette période est surtout marquée par

1 Th. S BALIMA, La radiodiffusion en Haute-Volta : système institutionnel et évolution soci-culturelle, 1980.

2 D. BAGOYOKO, L'économie des moyens d'information au Mali, 1987.

3 A. POUYA, Die Deutsche Welle : la voix de l'Allemagne et ses auditeurs "francophones » d'Afrique, 1988.

voir aussi E Tjadè, Radios, publics et pouvoir au Cameroun - utilisation officielle et besoins sociaux, L'Harmattan, 1986.

S. B. TCHIKAYA, Les relations entre RFI et les pays de l'Afrique centrale francophone 1978-1980, 1984.

4 Th. E. VITTIN, Les radios internationales en Afrique noire, 1995. et « Les radios internationales, acteurs de la vie politique en Afrique noire », 1997.

Voir aussi M. Samb, Etude de la radio au Sénégal et des nouvelles stratégies des stations internationales, 1996.

5 PANOS, Le pluralisme radiophonique en Afrique Subsaharienne, 2 vol., 1993.

6 E TJADÈ Démonopolisation, libéralisation et liberté de communication au Cameroun, L'Harmattan, 2001. E.A.K Atchikiti., Les radios privées dans le paysage 
l'introduction de questions nouvelles : radio et démocratie ${ }^{1}$, la liberté d'expression ${ }^{2}$, radio et citoyenneté ${ }^{3}$, la place des radios d'États dans le nouveau paysage radiophonique, le rôle des instances de régulation... Un certain nombre de travaux se sont par ailleurs orientés vers une mise en perspective historique, car si dans beaucoup de pays l'histoire de la radio existe, pour nombre d'entre eux, ce n'est pas encore le cas.

Ces derniers temps, on se tourne de plus en plus vers le rôle de la radio dans une situation de crise. L'expérience de la radio des mille collines a fait redécouvrir la radio comme une arme et une arme à double tranchant. Le risque qu'elle ne soit mal utilisée en temps de conflit inspire un certains nombre de réflexions. L'autre thème majeur actuellement objet d'étude sur la radio en Afrique est le phénomène de radios communautaires et de radios de proximité.

\section{Problématiques et pistes de cherches sur les radios africaines}

Pour beaucoup de pays africains, on ne peut pas dire que la radio a été insuffisamment étudiée. Il faut reconnaître d'ailleurs de façon générale que le retard à l'échelle de tout le continent n'est que relatif. Cependant, au regard de l'évolution rapide du sujet entraînant les mutations à tous les niveaux, il nécessite de nouvelles postures heuristiques, herméneutiques, épistémologiques et méthodologiques ainsi que de nouveaux questionnements. Sans vouloir prétendre à en fournir, nous voudrions cependant faire quelques suggestions en termes de pistes de recherches, indiquer les sources et éventuellement signaler quelques points en matière de méthodes d'investigation.

\section{Problématiques spécifiques}

La radio en Afrique pose des problèmes spécifiques en plus des questions générales dans lesquelles elle se situe. Tout en reprenant les

audio-visuel togolais, 2000.

1 RAYMONDO K.D., Le rôle des médias dans le processus démocratique au Togo, 1993.

2 N.N. OUENDJI, Radios privées et liberté d'expression au Cameroun, 2004.

3 Sylvie CAPITANT, Radio et citoyenneté au Burkina faso, Une thèse en cours. 
thèmes classiques des études des médias, il faudra intégrer des données spécifiques et parfois poser les questions autrement. Eu égard à la situation actuelle des radios en Afrique, il y a de nombreuses voies à explorer.

Une première voie pourrait prolonger la réflexion entamée dans les années 1980-1990 sur les fonctions de la radio pour permettre de mieux appréhender l'impact et le rôle des radios dans les sociétés africaines, leurs incidences politiques et culturelles, leur contribution effective au développement, à la démocratie et à la citoyenneté ainsi que l'emprise réelle de la radio dans la vie quotidienne.

Une seconde voie pourrait s'organiser autour des questions touchant aux mutations récentes en matière de statut juridique, de conditions d'exploitation, de financement.

Une troisième piste pourrait s'orienter vers la radio du futur ! Même si à cause de la fracture numérique l'A frique reste relativement en marge du développement des nouvelles technologies, elle connaît le transfert de ces dernières sur son terrain. Ainsi les questions nouvelles autour des radios numériques, du postcasting, etc. pourraient se poser aussi déjà pour l'Afrique. Il faudra observer les diverses pratiques radiophoniques qu'induit l'avènement de ces nouvelles technologies.

La communication religieuse est en passe de devenir une option préférentielle des radios africaines. Quels publics, quels contenus, quelles fonctions, quels enjeux, quelles influences ? ${ }^{1}$

L'étude des publics, des audiences, de la réception qui sont pourtant des thèmes classiques font aujourd' hui défaut en Afrique. Il n'y a pas de public homogène et la multiplicité des radios ne permet pas de se faire facilement une idée sur les audiences des radios et des programmes.

L'oralité pourrait constituer également un autre champ d'investigation. Avec les mutations incessantes et vertigineuses des pratiques radiophoniques en Occident, peut-être l'Afrique nous offrira-t-elle la chance de conserver pour un temps encore la réalité de « radio pure nature ».

1 Nous en avons fait depuis quelques années le centre d'intérêt de notre recherche : 1. «les causes de l'engouement des populations de Lomé pour les radios privées », 2002 ; 2. « Radios et fait religieux en Afrique, contenu, fonctions et enjeux. Le cas du Togo, 2004 ; 3. «Les radios chrétiennes en Afrique et la spiritualité missionnaire : véhicules ou lieux d'élaboration? », 2006 ; 4. thèse en cours : « Radios et Religion en Afrique ». 
D'autres questions spécifiques touchant par exemple la place croissante de la femme dans la pratique radiophonique, les radios de proximité...

Il s'agit de déplacer le problème des radios en Afrique des poncifs et autres lieux communs sur celui des enjeux et des défis (technologiques, médiatiques, politiques et culturels).

\section{Méthode spécifique}

Pour une multitude de raisons, le terrain africain présente toutes sortes de particularités. Elles font que les méthodes et les outils de mesure, d'observation et d'analyse élaborés dans les laboratoires occidentaux ne s'adaptent que difficilement. Il faut donc avoir à l'esprit de tenir compte du milieu et du contexte (histoire, culture, environnement sociopolitique, économique, etc.) à tous les niveaux de la démarche. Si l'on veut par exemple étudier l'audience des radios, les trois moments (1- le parc récepteur, 2- les postes émetteurs et la durée d'écoute, 3- la réception et l'influence de la radio) sont difficilement applicables. Le parc radiophonique acquiert une importance moindre à cause de la difficulté à évaluer les entrées et l'usage individuelle des postes récepteurs. Le nombre d'heures d'écoute est difficilement mesurable à cause de l'élasticité de la notion du temps. En ce qui concerne la réception, l'analyse de l'attention, de la compréhension, la perception des messages suppose la mémorisation ou la consignation par écrit que l'analphabétisme encore large ne favorise pas.

Il serait ensuite souhaitable que les réflexions se fassent au niveau général. Il existent peu d'étude d'ensemble, la plupart de celles existantes se circonscrivant préférablement à un pays ou à une région. Les nombreuses monographies réalisées peuvent à présent permettre des perspectives comparatives à l'échelle de tout le continent. On a certes beaucoup de différences d'un pays à l'autre mais sur le plan des médias, les similitudes sont plus grandes que les particularités.

La diversité des aspects et des thèmes appelle une implication de plusieurs disciplines. Les sciences de l'information et de la communication avec une touche d'anthropologie, la sociologie, l'histoire, les sciences politiques, la géographie, la linguistique, etc. pourraient y trouver matière à étudier. 
Cela nécessitera également un investissement collégial à travers un ou plusieurs groupes de recherches exclusivement concentrés sur la radio. La concertation autour des méthodes d'investigation et d'analyse ainsi que la mise en commun des différentes sources éparpillées ici et là devraient mobiliser une véritable synergie.

\section{Sources et ressources des études des radios africaines}

La question des sources d'information sur les médias africains est un vrai problème. Ils ont été introduits en Afrique pendant la période coloniale et étudiés par la suite surtout par les occidentaux, la plupart des informations qui s'inscrivent dans une logique historique se situent dans les capitales occidentales (Londres, Paris, Lisbonne, etc.). Même les travaux les plus récents ne sont diffusés en Afrique que très partiellement. En matière d'études, on ne trouvera sur place que des sources de moindre importance.

\section{Sources historiques}

À l'exception de l'Afrique du Sud et, dans une certaine mesure le Sénégal, qui disposent d'une documentation sur les premières radios africaines, pour les autres pays, les sources concernant les différents aspects de la radiophonie (structures, contenu, audiences) jusque dans les années 1970-1980, sont en Occident. C'est d'abord dans les rapports des ministères ou des services concernés par la radiodiffusion qu'on trouve les informations sur les débuts de la radio, sur les structures juridico-politiques, le financement, les questions techniques, etc. À Londres par exemple la documentation accumulée par le British Colonial Office Committee, le Conolial Office. À Ottawa, le Département de la Communication a aussi des archives sur l'A Arique.

On les trouve ensuite dans les archives des radios mères des métropoles : la BBC (BBC Handbook), la Société de Radiodiffusion de la France d'Outre-Mer (SOFORAM), etc.

On trouve également des informations dans les archives d'organismes de certains États Occidentaux : la United States Information Agency (USIA) à New York a produit pendant un certain nombre d'an- 
nées un rapport annuel sur les radios pays par pays, l'International Radio Consultative Commitee (CCIR) dispose ainsi à Genève de données sur les radios africaines. La revue annuelle de la Canadian International Developement Agency qui a formé beaucoup de journalistes africains et a financé la construction des écoles de journalismes sur le Continent comporte aussi des informations sur l'Afrique ainsi que le Rundfunk und Fernsehen de Hambourg en Allemagne. Signalons enfin dans ce domaine le rapport annuel de l'UNESCO à Paris ainsi que plusieurs études commanditées par cet organisme.

On trouve par ailleurs des revues et ouvrages : World RadioTelevision Handbook, Journal of Broadcasting, International and comparative Broadcasting qui a publié plusieurs études comparatives des radios africaines dont celles de Sydney W. Head en 1974 sous le nom de «Broadcasting in Africa, a continental survey of Radio and Television » qui fourmille de toutes sortes d'informations sur la radio dans chaque pays et sur des questions d'ensemble touchant l'aide bilatérale, les radios internationales, les programmes religieux, la formation, etc.

Les informations sur les audiences sont à trouver auprès des organismes (SCOPE en Suisse pour les besoins commerciaux, USIAD aux États-Unis d'Amérique pour le développement, l'UNESCO pour le développement et la culture etc. ou des radios Internationales (BBC, RFI, VOA, DW) qui les ont commanditées, ou auprès des sociétés de sondages (IFOP, MARCOMER, SOFIRES).

Plusieurs universités en Europe et en Amérique du Nord disposent également dans leurs bibliothèques de thèses et d'études sur l'Afrique.

\section{Le présent}

Les études sont grandement facilitées par Internet; il y a beaucoup de site web de radios. On peut les écouter chez soi sans être obligé d'aller sur place, quand par exemple on veut faire une simple analyse de contenu des programmes. Malheureusement ces sites ne durent pas longtemps ou ne sont souvent pas actualisés. Il y a également des sites des associations de radios communautaires et associatives, de radios commerciales, des sites d'associations de professionnels, etc. 
Rien ne remplacera cependant le terrain. Et dans ce sens, l'Afrique ou plutôt les Afriques offrent une réalité particulière. On ne fera jamais assez noter les difficultés liées à la localisation des informations, à leur collecte, etc. On trouvera quand même sur place, outre les archives sonores dans les maisons de radios, les mémoires d'étudiants dans les bibliothèques universitaires, des documents officiels sur le statut juridique, les conditions d'exploitation, les cahiers de charges, etc. dans les instances de gestion et de contrôle des médias.

\section{Conclusion}

La radio est un sujet très mouvant. Cela est vrai en Occident avec l'avènement des nouvelles technologies de l'information et de la communication engendrant de nouvelles pratiques. Mais cela est vrai aussi pour l'Afrique pour ces mêmes raisons et pour d'autres encore liées à l'évolution constante des sociétés africaines. Les mutations rapides subies par la radio ces dernières décennies par suite de changements politiques et de la globalisation de la communication méritent d'être évaluées avant que les repères ne se brouillent. Cela demande une mobilisation des chercheurs africains qui devront s'organiser dans un contexte d'absence totale de financement de la recherche s'il veulent que le travail soit efficace. Mais un regard extérieur d'Africanistes peut être utile pour enrichir le débat. Le champ est vaste, il y a du travail pour tout le monde. 